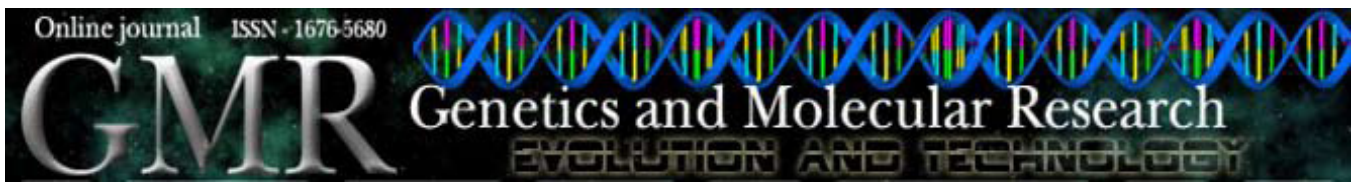

Review

\title{
Jasmonates are phytohormones with multiple functions, including plant defense and reproduction
}

\author{
N.C. Avanci ${ }^{1,2}$, D.D. Luche ${ }^{1}$, G.H. Goldman ${ }^{3}$ and M.H.S. Goldman ${ }^{1}$ \\ ${ }^{1}$ Departamento de Biologia, Faculdade de Filosofia, \\ Ciências e Letras de Ribeirão Preto, \\ Universidade de São Paulo, Ribeirão Preto, SP, Brasil \\ ${ }^{2}$ Departamento de Biologia, PPG Biologia Comparada, \\ Faculdade de Filosofia, Ciências e Letras de Ribeirão Preto, \\ Universidade de São Paulo, Ribeirão Preto, SP, Brasil \\ ${ }^{3}$ Departamento de Ciências Farmacêuticas, \\ Faculdade de Ciências Farmacêuticas de Ribeirão Preto, \\ Universidade de São Paulo, Ribeirão Preto, SP, Brasil \\ Corresponding author: M.H.S. Goldman \\ E-mail: mgoldman@ffclrp.usp.br
}

Genet. Mol. Res. 9 (1): 484-505 (2010)

Received December 4, 2009

Accepted January 10, 2010

Published March 16, 2010

\begin{abstract}
The plant hormones jasmonic acid and methyl jasmonate, along with their intermediate compounds, produced in the octadecanoid pathway, are important signaling molecules that are collectively called jasmonates. These are widespread in the plant kingdom and play crucial roles in biotic/abiotic stress responses, as well as in processes related to plant growth and development. Recently, it has been shown that jasmonates are also involved in reproductive processes. We present the most recent findings related to the biosynthesis, regulation and signaling mechanisms of jasmonates. Additionally, we discuss the identification of [(+)-7-iso-JA-L-Ile] as the active biological hormonal form of jasmonate; this fills the greatest gap in our knowledge about the signaling mechanism that is responsible for the activation of downstream genes in the jasmonate-signaling cascade. The identification of several
\end{abstract}


Arabidopsis thaliana mutants was crucial to the elucidation of the signaling mechanisms involved in jasmonate-mediated responses. Finally, the involvement of jasmonates in the reproductive process of Nicotiana tabacum L. is briefly discussed, since some of the main enzymes of the jasmonic acid biosynthesis pathway were identified in a stigma/style expressed sequence tag database (TOBEST) of this Solanaceae species.

Key words: Jasmonic acid; Methyl jasmonate; Signaling; Reproductive process; Defense response; Nicotiana tabacum L.

\section{INTRODUCTION}

As sessile organisms, plants need to produce a huge arsenal of chemical compounds, both volatile and non-volatile, to adapt and respond to environmental challenges. Among these compounds, the phytohormones are in a prominent position, playing important regulatory roles in plant physiology (Wasternack and Hause, 2002; Chen et al., 2006; Browse, 2009a). During the past 20 years, a class of phytohormones derived from the metabolism of membrane fatty acids, collectively known as jasmonates, has attracted considerable attention. Jasmonic acid (JA), methyl jasmonate (MeJA), 12-oxophytodienoic acid (12-OPDA), JA conjugated to some amino acids such as leucine (JA-leucine) and isoleucine (JA-isoleucine), among other jasmonates, are widespread in the plant kingdom. These compounds are involved in crucial processes related to plant development and survival, including direct and indirect defense responses, secondary metabolism, reproductive process, senescence, fruit development, and tritrophic interactions (Seo et al., 2001; Arimura et al., 2005; Liechti and Farmer, 2006; Wasternack, 2007).

First identified and isolated from a culture of the fungus Lasiodiplodia theobromae, JA is the best-known and best-characterized member of the jasmonate family. Recent studies have shown that JA can be modified in various ways, such as conjugation to different amino acids or hydroxylation, forming compounds of extreme importance for the activation of jasmonate-responsive defense genes (Creelman and Mullet, 1995; Liechti and Farmer, 2006; Thines et al., 2007). In addition, it has also been shown that JA plays crucial roles in reproduction of both Arabidopsis (Sanders et al., 2000; von Malek et al., 2002) and tomato plants (Hause et al., 2000; Li et al., 2001, 2004).

Besides JA, another jasmonate of key importance is its methyl ester form, MeJA. First isolated from essential oil extracted from Jasminum grandiflorum petals, due to its volatile property, MeJA initially aroused considerable commercial interest from the perfume industry, stimulating studies focused on its structure and synthesis. During the 1980's the first reports of physiological effects attributed to JA and MeJA appeared, describing activities related to senescence in Artemisia absinthium (Ueda and Kato, 1980), and growth inhibition in Vicia faba (Dathe et al., 1981). Since then, the number of metabolic and physiological activities attributed to jasmonates continues to increase. Due to its volatile nature and ability to diffuse through membranes, MeJA has been considered an important candidate for an airborne signal molecule mediating intra- and interplant communications, modulating plant defense responses (Demole et al., 1962; Creelman and Mullet, 1995; Seo et al., 2001; Wasternack, 2007) and regulating plant reproductive processes (von Malek et al., 2002).

Here, we examine the biosynthesis and metabolism of jasmonates, focusing on 
defense responses and reproductive processes in plants. Furthermore, we put into context the great importance of discovering the authentic biologically active hormonal form of the jasmonate-signaling mechanism: [(+)-7-iso-JA-L-Ile]. Additionally, we examined the possible involvement of jasmonates in the Nicotiana tabacum reproductive process. For more detailed information concerning the metabolism, function and signaling network of jasmonates, recent papers and reviews should be consulted (Berger, 2002; Farmer et al., 2003; Halitschke and Baldwin, 2004; Lorenzo and Solano, 2005; Delker et al., 2006; Wasternack, 2007; Kazan and Manners, 2008; Browse, 2009a; Fonseca et al., 2009; Yi et al., 2009).

\section{Jasmonate biosynthesis}

In a pioneer research, Vick and Zimmerman (1984) were the first authors to demonstrate the steps of the JA biosynthesis pathway in a detailed manner. Since then, jasmonate biosynthesis and signaling pathways have been extensively studied, mainly in dicots such as Arabidopsis (Arabidopsis thaliana) and tomato (Solanum lycopersicum), and to a lesser extent in some monocots (Kazan and Manners, 2008).

The jasmonates JA, MeJA and their octadecanoid precursors (e.g., 12-OPDA) are lipidic molecules with a great capacity to regulate diverse physiological processes. They are produced from $\alpha$-linolenic acid ( $\alpha$-LeA, 18:3) present in chloroplast membranes (Figure 1). These compounds, also known as oxylipins, are similar in structure and biogenesis to prostaglandins, important signaling molecules in invertebrates and vertebrates (Cheong and Choi, 2003; Kazan and Manners, 2008). Formation of $\alpha$-LeA from lipids present in chloroplast membranes occurs through the action of type A phospholipases and is triggered by external stimuli, such as wounding or pathogen attacks. Upon release into the chloroplast interior, a molecule of oxygen is added to $\alpha$-LeA, in a reaction catalyzed by 13-lipoxygenase (LOX), forming 13-hydroperoxy-9,11,15-octadecatrienoic acid (13-HPOT). This is considered to be the initial step in JA formation by the LOX pathway. The 13-HPOT is oxidized by allene oxide synthase (AOS), forming an allene oxide that is chemically unstable, which is converted to 12-OPDA by the enzyme allene oxide cyclase. Laudert and Weiler (1998) reported that in A. thaliana the gene $A O S$ contains a signal sequence for the chloroplast, and it is present as a single copy gene in the genome; thus, it is considered to be fundamental for the regulation of JA biosynthesis.

12-OPDA is the immediate precursor of JA and is the last product of the metabolic pathway of JA biosynthesis that occurs in the chloroplast. For the final steps in JA production, the 12-OPDA molecule undergoes three cycles of $\beta$-oxidation (Figure 1) inside the peroxysomes; this is the only organelle known to be able to perform such reactions in plants. There is evidence that 12-OPDA transport to the peroxysomes is partially dependent on ABC COMATOSE in $A$. thaliana (Rosahl and Feussner, 2005; Delker et al., 2006; Liechti and Farmer, 2006; Browse, 2009a). Once formed, JA may go through different metabolic conversions originating distinct jasmonates. Methylation reactions catalyzed by JA methyltransferases will produce MeJA, a reaction believed to occur in the cytoplasm (Seo et al., 2001).

\section{Mutants and transgenic plants have contributed to unravel the multiple jasmonate functions}

In A. thaliana, the CEV1 gene encodes a cellulose synthase that is capable of syn- 


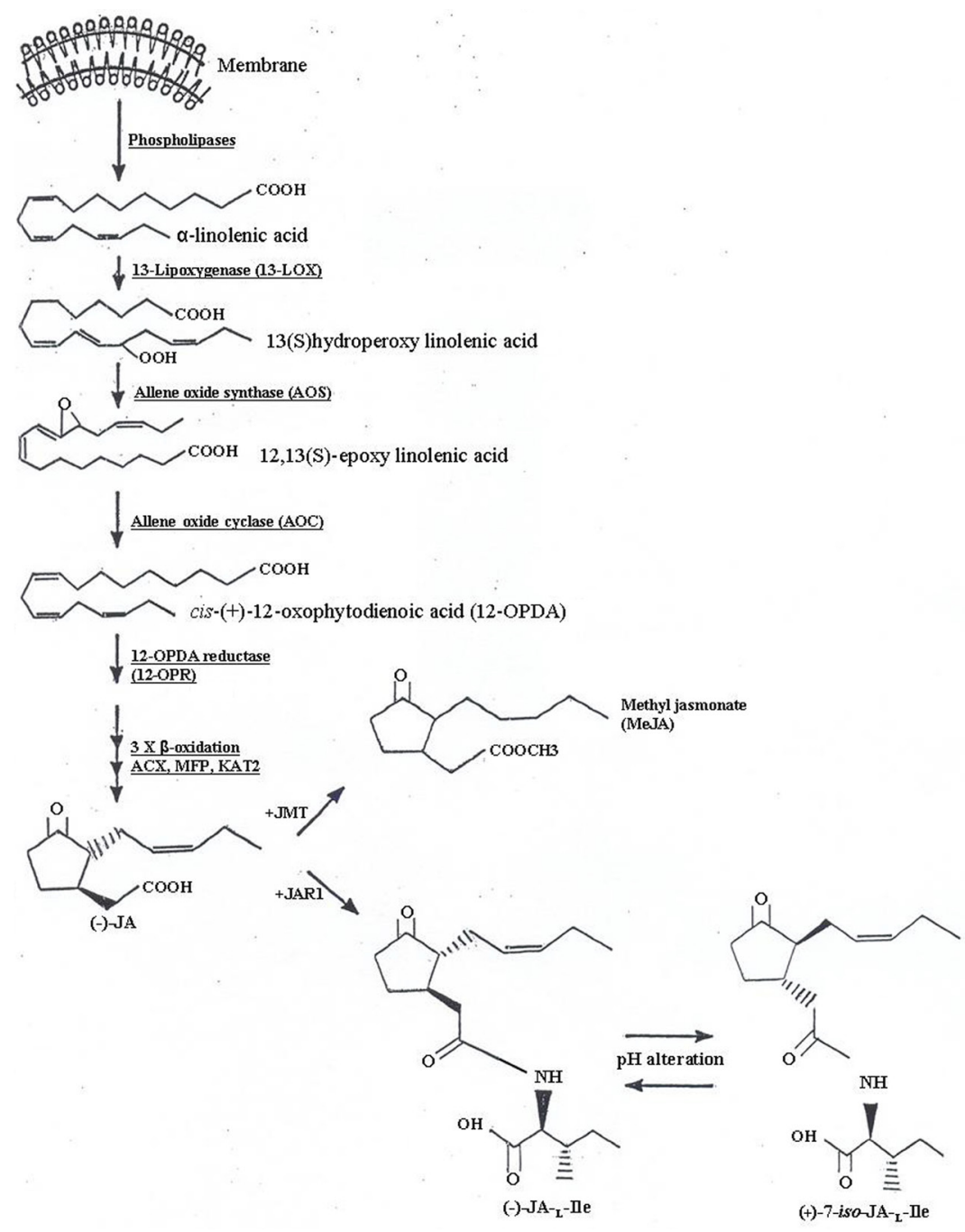

Figure 1. Schematic representation of the jasmonic acid (JA) biosynthetic pathway, including all intermediate oxylipins (modified from Wasternack, 2007 and Browse, 2009a). The main enzymes are in bold and underlined. Synthesis begins with $\alpha$-linolenic acid released from the chloroplast membranes, after the action of phospholipase enzymes. At the end, the 12-oxophytodienoic acid (12-OPDA) molecule is translocated to peroxisomes, where it undergoes three $\beta$-oxidation steps, leading to the formation of JA. This synthesis scheme is based on studies of Arabidopsis thaliana plants.

thesizing cellulose in root tissues. Mutants of the cevl gene produce less cellulose in roots when compared to wild-type plants; these mutants overexpress the VSP, THI2.1, PDF1.2, and $C H I-B$ genes, known to constitutively accumulate in response to jasmonate and ethylene signaling (Ellis et al., 2002). Accordingly, cev1 mutants have increased jasmonate and ethylene production; suppression of this phenotype is obtained by mutations that cause interruption of 
the signaling pathways of the hormones. This demonstrates that modifications in the cell wall can trigger stress responses in plants (Ellis et al., 2002).

Maciejewska and Kopcewicz (2003) demonstrated the contribution of jasmonate towards control of growth and elongation in shoot and root tissues, as well as in the formation of floral buds in Pharbitis nil, known as Japanese Morning Glory. When 4-dayold seedlings were incubated in solutions containing MeJA, growth of primary roots was notably inhibited: the higher the MeJA concentration, the greater the inhibitory effect, reaching $83 \%$ with a $1 \mathrm{mM}$ concentration. MeJA application through the roots inhibited the formation of floral buds when the treatment was performed before or during photoinduction. Plants treated during these periods produced 3- to 4-fold less floral buds than control plants. It was also observed increasing periods of incubation with MeJAincreased inhibition of floral bud formation. Taken together, these results demonstrate the involvement of MeJA in flowering, growth and elongation of shoots and roots in $P$. nil. No correlation, direct or indirect, was observed between inhibition of growth and inhibition of flowering, indicating that MeJA acts independently in the control of these two processes (Maciejewska and Kopcewicz, 2003).

The contribution of jasmonates to morphogenetic events is well documented. Besides promoting cell expansion, these phytohormones can control cell division and growth direction, supporting the adequate formation of tissues and organs (Koda, 1997). NTR-1 encodes a methyltransferase capable of using JA as a substrate to produce MeJA through a methylation reaction (Song et al., 2000). Transgenic soybean plants expressing the NTR-1 gene from Brassica campestris under the control of the $35 \mathrm{~S}$ promoter showed alterations in leaf and root development. These plants, with an increased endogenous MeJA level, exhibited leaves that were slightly elongated and considerably narrowed compared to the ones of wild-type plants (Xue and Zhang, 2007). There was also an inhibitory effect on primary root elongation and a stimulatory effect on lateral root development. Concentrations of MeJA in the leaves of these transgenic plants were 2- to 2.5 -fold higher than untransformed control plants. This shows that increased endogenous MeJA levels promote visible alterations in the regulation of morphogenesis in soybeans (Xue and Zhang, 2007).

It is known that jasmonates have a central role in the regulation of the biosynthesis of several secondary metabolites, including terpenoids, alcaloids, phenylpropanoids, and antioxidants. The constitutive activation of the jasmonate-signaling pathway causes enhanced production of secondary metabolites in tomato plants. Exogenous application of MeJA promotes accumulation of caffeoylputrescine in tomato leaves, a metabolite derived from the phenylpropanoid and polyamine pathways; it has a putative function in the plant reproductive process (Chen et al., 2006). Mutants of jail, insensitive to jasmonate signaling, do not accumulate caffeoylputrescine in flowers and leaves treated with MeJA. On the other hand, a transgenic tomato line, overexpressing prosystemin, a signal molecule involved in the local and systemic responses to pathogen attack, exhibited high levels of caffeoylputrescine in leaves not treated with MeJA. RNA blot analyses showed that caffeoylputrescine biosynthesis was up-regulated in wild-type plants treated with MeJA and in ProSystemin (PS) transgenic plants. Consequently, accumulation of caffeoylputrescine in tomato leaves is affected by jasmonate signaling (Chen et al., 2006).

Very little is known about the jasmonate-signaling pathway in monocots, including those of great economic importance, such as corn and rice. Jasmonates are involved in the activation of genes related to oxidative stress in maize (Norastehnia and Asghari, 2006) and in the expression of 
genes for resistance against attack by fungal pathogens in rice (Mei et al., 2006). Maize seedlings were treated with Paraquat, an herbicide widely used in agriculture, in combination with MeJA. Different MeJA concentrations $(0,5,10,20,50$, and $100 \mu \mathrm{M})$ were applied to maize plants before and after the herbicide treatment and the expression of genes corresponding to the anti-oxidative defense system investigated in shoots and roots. It was found that MeJA (50 and $100 \mu \mathrm{M})$ promoted increased production of several anti-oxidative enzymes, including glutathione reductase, guaiacol peroxidase and ascorbate peroxidase. The physiological mechanisms involved in the increase of these enzymatic activities mediated by MeJA are still unknown. However, it has been suggested that this increase may be due to up-regulation of genes controlling the synthesis of these enzymes, or by activation of diverse constitutive genes (Norastehnia and Asghari, 2006). Besides mediating abiotic stress responses, jasmonates also have modulator activities related to biotic stresses. Rice transgenic plants constitutively expressing the AOS enzyme, which is an extremely important enzyme in the JA biosynthetic pathway, showed increased resistance to attack by pathogenic fungi and a robust induction of pathogen-related genes, such as PRIa, PR3 and PR5 (Mei et al., 2006). Additionally, exogenous JA application in rice seedlings activated the expression of genes related to defense and induced local resistance against the fungus Magnaporthe grisea. When compared to wild-type plants, the transgenic lines had increased levels of endogenous JA, mainly after infection with $M$. grisea. Taken together, these results clearly demonstrate the functionality of the $A O S$ gene affecting the JA biosynthetic pathway in monocots (Mei et al., 2006).

The LOX enzyme is known to fulfill an important role at the beginning of the jasmonate synthesis cascade, by adding molecular oxygen to $\alpha$-LeA (Hause et al., 2000). Transgenic Nicotiana attenuata plants expressing the $L O X 3$ gene in the antisense orientation had decreased accumulation of damage-induced JA, reducing resistance to herbivory. Mechanisms of indirect defense were also affected, since release of the volatile terpenoid compound, capable of attracting generalist predators that feed on herbivores, was drastically reduced (Halitschke and Baldwin, 2003). Well-known mechanisms of direct defense induced by herbivores, such as nicotine and trypsin protease inhibitors, were also greatly reduced in these transgenic plants. However, exogenous treatment with MeJA was able to completely reestablish all the phenotypes mentioned above, with the exception of trypsin protease inhibitor activity, which was only partially reestablished. These data demonstrate that compounds produced in steps before LOX3 in the JA biosynthesis pathway have a fundamental role in the up-regulation of defense-related genes in N. attenuata (Halitschke and Baldwin, 2003).

The articles revised here show that there is a vast diversity of physiological processes that are regulated by the jasmonate signaling pathway. All these processes are extremely important for the survival of plants that are constantly submitted to many environmental challenges. Most of the time, such challenges need a quick physiological response to allow plants to survive and reproduce with success in their natural environment. Our understanding concerning the potential roles of jasmonates in plants is rapidly expanding (Kazan and Manners, 2008); nevertheless, there is still much to be learned about the processes regulated by the jasmonate pathway, as well as concerning its own regulation.

\section{Jasmonate signaling: a hormonal mechanism involving repressor molecules, transcription factors and proteasomal degradation}

Here we summarize some of the most recent findings about regulatory mechanisms involving jasmonate-mediated responses. These responses point to a complex interaction be- 
tween different molecules, such as regulatory proteins, transcription factors, ubiquitin-mediated protein degradation in proteasomes, as well as jasmonates in their bioactive forms.

Once synthesized in the peroxisomes, JA is probably rapidly recognized and transduced by specific receptors within the cells (Beckers and Spoel, 2006). In spite of considerable effort trying to identify these receptors, their localization inside the cells remains unknown (Wasternack, 2007). Currently, there is considerable evidence supporting the idea that after binding to receptors, jasmonates trigger a signal-transduction pathway capable of either activating or repressing JA-regulated genes (Kazan and Manners, 2008). A large part of the knowledge regarding JA action comes from screening collections of Arabidopsis mutants that are unable to either synthesize or respond to JA (Thines et al., 2007). The ability of JAs to inhibit root growth during plant development has been widely explored in order to identify mutants in this pathway. As a result, the coronatine-insensitivel (coil) was one of the first mutants identified in the jasmonate pathway in Arabidopsis (Feys et al., 1994; Beckers and Spoel, 2006; Kazan and Manners, 2008). Seedlings of coil had reduced sensitivity for root elongation when treated with JA and coronatine, a phytotoxin structurally and functionally similar to JA, produced by the pathogenic bacteria Pseudomonas syringae (Feys et al., 1994). Additional analysis showed that coil also fails to complete important physiological responses that are dependent on JA, such as fertility and secondary metabolite biosynthesis. These mutants are also highly susceptible to insect infestation and to pathogen infection, suggesting a central role for COII in the regulation of JA-mediated signaling (Beckers and Spoel, 2006; Kazan and Manners, 2008). The COII gene codes for a $66-\mathrm{kDa}$ protein, bearing an N-terminal region containing an F-box motif and 16 leucine-rich repeats. F-box proteins form multi-protein complexes that work as receptors, specifically recruiting regulatory proteins as substrates, directing them to degradation pathways mediated by ubiquitin-proteasome $26 \mathrm{~S}$ complexes. For this reason, COII has been suggested to effectively participate in removing repressors from the JA-signaling cascade (Figure 2) (Xie et al., 1998). Moreover, the recruitment of such repressors is mediated by SCF complexes, composed of the following proteins: SKP1, cullin, and an F-box protein responsible for its substrate specificity (Beckers and Spoel, 2006). Recent studies have demonstrated that COI1 interacts directly with SKP1 and cullin in plants, supporting the hypothesis that COI1 is a component of the $\mathrm{SCF}^{\mathrm{COII}}$ ubiquitin-ligase complex. RNAi transgenic plants that have diminished expression of one of these components, display reduced expression of JA-regulated genes, demonstrating the functionality of this complex (Xu et al., 2002). Additionally, COI1 interacts with a histone deacetylase, a protein that could be involved in repression of transcription, further strengthening the idea that COII derepresses the activation of JA-regulated genes, by targeting repressor proteins to ubiquitination and subsequent degradation (Devoto et al., 2002).

$\mathrm{Li}$ and co-workers (2004) described the molecular and phenotypic characterization of a sterile mutant in tomato (Solanum lycopersicum) plants insensitive to the JA-signaling pathway, named jasmonic acid-insensitivel (jail). Based on the jail-deduced amino acid sequence, which shows $68 \%$ identity with the Arabidopsis COI, it was demonstrated that JAI1 is a tomato COI1 homolog. Furthermore, as in Arabidopsis (Xie et al., 1998), tomato COI1-homolog protein possesses a conserved F-box motif and 16 leucine-rich repeats at the N-terminal portion. Hence, jail plants present a phenotype similar to Arabidopsis coil, as concerns the JA-signaling pathway. Defense-related responses are compromised due to the incapacity to activate the expression of JA-responsive genes, favoring attack by two-spotted spider mites. These plants also show reduced pollen viability and a sterile phenotype, which is caused by the incapacity to accumulate 


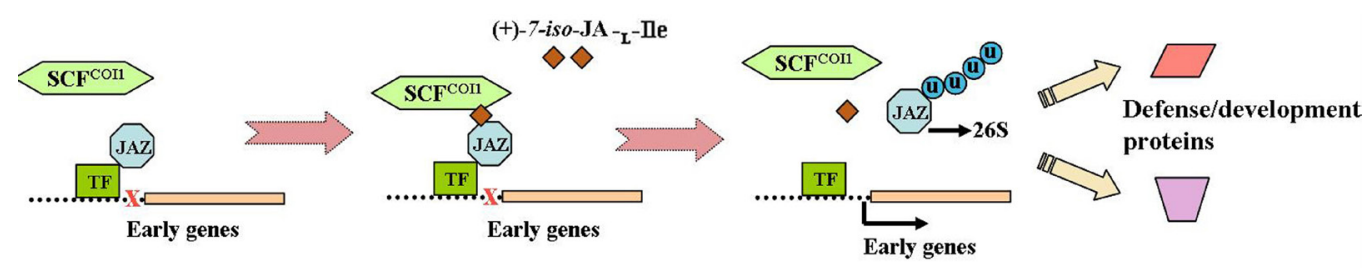

Figure 2. Simplified model of the molecular events that occur during the jasmonate-signaling pathway, according to Chini et al. (2007), Thines et al. (2007) and Fonseca et al. (2009). In the absence of external/internal stimuli (signals), JAZ proteins repress transcription factors (TF) capable of coordinating the activation of jasmonateregulated genes. However, in the presence of (+)-7-iso-JA-L-Ile, a jasmonate conjugate, the COI1 protein of the $\mathrm{SCF}^{\mathrm{CO} 11}$ complex interacts physically with JAZ proteins, leading the latter to be labeled with ubiquitin molecules. When the interaction between JAZ proteins and TF is disrupted, the jasmonate-response genes start to be expressed, while the JAZ proteins are targeted to the 26S-proteasome for subsequent degradation.

JA-regulated proteinase inhibitors, leading to the loss of seed maturation control. Mutant jail plants also have abnormal development of glandular trichomes of leaves, sepals, and green fruits, suggesting involvement of the JA-signaling pathway in defense responses related to glandular trichomes in tomato, implying that the JA/COI1 interaction signals distinct developmental processes in different plant species (Li et al., 2004). Similar to COI1,jarl was identified and isolated in a screening for mutants insensitive to JA and that had root growth inhibition induced by MeJA (Staswick et al., 1992). jarl mutants are incapable of responding to JA, exhibit deeply diminished expression of JA-regulated genes, and are highly susceptible to pathogen attack (Staswick et al., 1998; Rosahl and Feussner, 2005). The protein encoded by the JAR1 gene belongs to a new class of enzymes (acyl-adenylate), endowed with the capacity of catalyzing activation of carboxylic groups in a vast number of substrates. Other studies have revealed that JAR1 protein is effective in specifically adenylating JA, suggesting that this modification is crucial for the signaling function of this molecule. Even more interesting was the demonstration that in vitro synthesized JAR1 was capable of conjugating JA and the amino acid isoleucine, which in turn inhibits root growth, revealing its biological activity as a signaling molecule (Staswick and Tiryaki, 2004).

Knowledge about jasmonate biosynthesis has increased enormously in the last few years (Liechti and Farmer, 2006; Wasternack, 2007; Browse, 2009a); however, knowledge about jasmonate signaling is not following at the same pace (Chini et al., 2007). It is known that JA-related signaling responses are directly associated with a major reset of gene expression of downstream genes in the pathway (Thines et al., 2007). After great efforts by many groups, two main steps were defined. The first involves components and regulators of the $\mathrm{SCF}^{\mathrm{COI1}}$ complex (Xie et al., 1998; Devoto et al., 2002; $\mathrm{Xu}$ et al., 2002). The second involves transcription factors, such as MYC2, which coordinate the expression of JA-regulated genes (Boter et al., 2004; Lorenzo et al., 2004). These findings raised the following hypothesis: activation of JA-mediated responses, involving transcription factors, requires ubiquitin-mediated protein degradation of a possible repressor, which is targeted to proteasome $26 \mathrm{~S}$ mediated by the $\mathrm{SCF}^{\mathrm{COI1}}$ complex, upon sensing jasmonates by plant cells (Figure 2) (Chini et al., 2007). Despite all the attempts to identify a possible target for the SCFCorl complex, all failed to reveal the candidate molecule (repressor), or even provide good clues about the connection between the $\mathrm{SCF}^{\mathrm{CO} I 1}$ complex and the entire transcriptome regulated by jasmonates (Browse, 2009a).

Two articles (Thines et al., 2007; Chini et al., 2007) elegantly described the identification of the $\mathrm{SCF}^{\mathrm{CO} I 1}$ complex target molecules in the jasmonate-signaling pathway. Thines and co- 
workers (2007) exogenously treated stamens of Arabidopsis opr 3 mutant (defective for jasmonate synthesis) with jasmonate; after 30 min significant induction of 31 genes was observed, when compared to control plants. Eight of them code for proteins with unknown function, seven of which possess a 28-amino acid conserved domain (ZIM domain); hence, these genes were named JASMONATE ZIM-DOMAIN (JAZ). They also reported that JAZ proteins fused to a GUS reporter gene; transgenic plants bearing the constructs $J A Z 1-G U S$ and $J A Z 6-G U S$ were obtained. Seedlings of the control plant carrying the GUS gene alone displayed intense blue color, especially in the root tissues. In contrast, the transgenic plants JAZ1-GUS and JAZ6-GUS exhibited a very faint signal when compared to controls. However, when the seedlings were treated with $100 \mu \mathrm{M} \mathrm{JA}$ for $1 \mathrm{~h}$, JAZ1-GUS and JAZ6-GUS plants have complete loss of GUS activity, while GUS activity was not affected in control plants. Consequently, it was suggested that jasmonates facilitate or intensify interaction between COI1 and JAZ proteins, and consequently they target JAZ protein for degradation by ubiquitination and proteosome $26 \mathrm{~S}$ degradation. Also, pull-down assays have shown that the JA-Ile conjugate is responsible for the physical interaction between COI1 (from the SCF complex) and JAZ proteins. Other jasmonate derivatives, including JA, MeJA and 12-OPDA, do interact. Likewise, pull-down assays with COI and JAZ from tomatoes demonstrated that COI1 and JAZ1 interact physically, in a specific manner, mediated by JA-Ile (Thines et al., 2007).

Another contribution relevant to our understanding of the regulatory mechanisms involved in the jasmonate-signaling pathway comes from the study of Chini et al. (2007). They identified the protein JASMONATE INSENSITIVE3 (JAI3) and demonstrated that it belongs to the JAZ protein family, the members of which are primary repressors of gene expression in the jasmonate-signaling pathway. They also showed that JAI3 and other JAZ proteins interact physically with COI1, and that exogenous treatment with jasmonates induces degradation by proteasome $26 \mathrm{~S}$, in a COI1dependent manner. Additionally, pull-down experiments demonstrated the physical interaction between JAI3 and MYC2, the main transcriptional activator for jasmonate responses. Moreover, wild-type and coil plants were transformed with the construction JAI3-GFP, and the degradation of JAI3 via proteasomes was also demonstrated. Fusion JAI3 proteins were detected in the nuclei of root cells in the absence of jasmonate treatment; they disappeared completely after treatment. Degradation of JAI3 was blocked in the presence of MG132, a specific inhibitor of proteasomes, demonstrating the participation of proteasome $26 \mathrm{~S}$ in the removal of JAI3 mediated by jasmonates. Treatment with jasmonate does not promote JAI3-GFP degradation in the coil mutant, clearly indicating that JAI3 is the target of the SCFCOI1 complex (Chini et al., 2007).

Taken together, these results demonstrate that binding of the hormone to its receptor induces degradation of JAZ proteins via proteasome $26 \mathrm{~S}$, in a manner that is dependent on the $\mathrm{SCF}^{\mathrm{COI1}}$ complex, releasing transcription factor $\mathrm{MYC}$, and allowing the transcriptional activation of response genes regulated by jasmonates. These results also demonstrate that JAZ/ JAI3 proteins are in fact the long-sought molecular link between the two main established steps in the jasmonate-signaling pathway (Thines et al., 2007; Chini et al., 2007).

Fonseca et al. (2009) unexpectedly found the jasmonate conjugate (-)-JA-L-Ile not to be the bioactive endogenous hormonal form, rather it was the isomer (+)-7-iso-JA-L-Ile. It is known that JA possesses four isomeric forms in nature (Sembdner and Parthier, 1993), and that the chemically synthesized commercial mixtures generally contain $90 \%$ of the stereoisomer (-)-JA-L-Ile and $10 \%$ (+)-7-iso-JA-L-Ile. Based on RP-HPLC analysis of their samples, 5-10\% were the isomer (+)-7-isoJA-L-Ile. Therefore, to answer which was the endogenous bioactive hormone, the four isomeric forms were synthesized, conjugated to the amino acid isoleucine, and then purified by SP-HPLC. 
After separation, the isomers (-)-JA-L-Ile and (+)-7-iso-JA-L-Ile were successfully purified and used in biological tests. Unexpectedly, the pure form (-)-JA-L-Ile did not possess detectable activity, albeit the (+)-7-iso-JA-L-Ile form was highly active at concentrations below $0.5 \mu \mathrm{M}$ in pull-down assays. This activity was even higher than that of the commercial mixture, and it was close to the activity of coronatine, the structural and functional homolog of the isomer (-)-JA-L-Ile. Also, pull-down experiments, confirmed by yeast two-hybrid assays, demonstrated that the interaction between COI1 and JAZ3 or JAZ9 is much stronger in the presence of (+)-7-iso-JA-L-Ile than with (-)-JA-L-Ile. Further analysis in Arabidopsis demonstrated that the (+)-7-iso-JA-L-Ile isomer is much more active than the previously proposed isomer. Remarkably, it was able to induce responses related to JA, such as inhibition of root growth, accumulation of anthocyanins, and efficient rescue of the jarl phenotype (insensitive to JA). This conclusively shows that the isomer (+)-7-iso-JA-L-Ile is the biologically active hormonal molecule in the jasmonate-signaling pathway (Fonseca et al., 2009). Chemical separation of isomers from commercial mixtures is tough; most likely a small percentage of (+)-7-iso-JA-L-Ile will remain in samples of (-)-JA-L-Ile. Though the pure isomer (-)-JA-L-Ile is less active than (+)-7-iso-JA-L-Ile and the coronatine molecule, (-)-JA-L-Ile can promote a biological response (Yi et al., 2009). A key point in finding (+)-7-iso-JA-L-Ile to be the bioactive form of the hormone was that Fonseca et al. (2009) dealt with purified forms of both isomers. Possibly, previous study describing (-)-JA-L-Ile as the bioactive form did not distinguish between the two molecules. In summary, the results prompted us to carefully review the literature about jasmonate-signaling pathways, especially regarding obtaining and preparing the material used in biological experiments. In any case, these new findings take us to a higher level of complexity with respect to the regulation of signaling pathways mediated by jasmonates.

\section{Jasmonates as molecular coordinators capable of orchestrating stress/defense responses in plants}

Known for their sessile nature, plants had to develop, along the evolutionary process, sophisticated ways of facing biotic and abiotic challenges in their natural habitats (Schilmiller and Howe, 2005). Two important mechanisms are: 1) production of wound-induced leaf compounds able to exert toxic effects, that have anti-nutritional properties, and 2) production and release of volatile compounds, eliciting indirect defense by attracting parasites and herbivore predators (Kessler and Baldwin, 2002). Plants are capable of a large array of responses, including cell wall reinforcement, synthesis of signaling compounds, eventual cell death, amongst others (Scheel, 1998). Jasmonates and their derivatives play a key role in activating genes involved in defense responses (Rosahl and Feussner, 2005) against abiotic stresses (drought, UV irradiation and ozone), and also against biotic stresses (infestation by insects and microbial pathogens). It is widely accepted that defense mechanisms, dependent on JA are activated upon infection by necrotrophic pathogens, which need to kill their host cells to gain access to nutrients (Rosahl and Feussner, 2005). The first detailed studies implicating the involvement of JA in wound-induced signaling and insect damage were made on Solanaceae. The discovery of woundinduced proteinases expressed in tomato (Green and Ryan, 1972) and potato (Sanchez-Serrano et al., 1986), along with the findings that expression of the corresponding genes was induced by exogenous JA treatment, were crucial for the establishment of a first study model for signaling triggered by wounds in plants (Farmer et al., 1992; Farmer and Ryan, 1992; Rosahl and Feussner, 2005).

Plants of the tomato mutant line JL5 were shown to be deficient in the activation of defense genes induced by wounding, hence named defenseless 1 (defl); they exhibit a compro- 
mised ability to accumulate JA when compared to wild-type plants. Additionally, the study of def1 mutant plants treated with intermediates of the octadecanoid pathway has demonstrated that this mutant is affected in octadecanoid metabolism between the synthesis of hydroperoxylinolenic acid (13-HPOT) and 12-OPDA. Furthermore, the mutant plants have a high susceptibility to the attack of the moth Manduca sexta (also known as the "tobacco hornworm"), demonstrating the key role played by the octadecanoid pathway in defense against insect attack (Howe et al., 1996).

LOX enzymes are fundamental at early steps in the biosynthesis of JA, and their involvement in defense responses has been reported in potato plants. The POTLX-3 gene identified in potato leaves, encodes an LOX enzyme, which has high sequence similarity with the tobacco LOX enzyme implicated in resistance mechanisms against the phytopathogen Phytophthora parasitica var. nicotianeae (Kolomiets et al., 2000). Healthy, undamaged, parts of potato plants do not accumulate POTLX-3 transcripts. Notwithstanding, potato leaves treated with ethylene or MeJA, or infected with virulent or non-virulent strains of Phytophthora infestans accumulate POTLX-3 mRNAs. In addition, an increase in POTLX-3 mRNA levels is observed during a hypersensitive response caused by the incompatible pathogen $P$ seudomonas syringae pv phaseolicola, suggesting the existence of an LOX pathway in potato leaves that is directly involved in defense mechanisms against pathogen attack (Kolomiets et al., 2000).

The involvement of the lipoxygenase pathway in defense responses was also demonstrated in cell suspensions of tobacco. When treated with an elicitor from P. parasitica var. nicotianeae, cells in suspension promote a fast and transient increase in the levels of JA, which stops after incubation with eicosatetraynoic acid, an inhibitor of LOX enzyme (Rickauer et al., 1997). Although pre-treatment with eicosatetraynoic acid subdues the induction of the proteinase inhibitors by the fungal pathogen, the same effect was not seen when cells were treated with MeJA. Though treatment with $\alpha$-LeA, a precursor in the biosynthesis of JA, induces defense responses by the cell suspension, linoleic acid did not achieve the same effect. Also, treatment with the pathogen, or MeJA, led to the expression of defense-related genes that code for type II proteinase inhibitors, glycoproteins rich in glycine and hydroxyproline, as well as glucanases and chitinases. Hence, synthesis of JA through the LOX pathway appears to be part of a complex regulatory mechanism involved in diverse defense responses (Rickauer et al., 1997).

Methylation reactions catalyzed by JA methyltransferases produce MeJA in A. thaliana (Seo et al., 2001). Nevertheless, overexpression of JA methyltransferase, leading to the accumulation of MeJA, did not alter the physiological levels of JA, despite increasing the resistance against pathogen attack in this plant. Hence, MeJA is suggested to be the active form of JA under specific physiological conditions (Seo et al., 2001; Delker et al., 2006).

Along the same line, MeJA was also shown to promote expression of defense-related genes in grapes (Belhadj et al., 2006). Grapevines attacked by fungal pathogens usually result in reduced production and quality of wine. In order to increase tolerance against such pathogens, grapevines (Vitis vinifera) were treated exogenously with MeJA. Treated leaves exhibited increased levels of transcripts coding for proteins related to pathogenicity, such as acidic class IV chitinase, serine protease inhibitor, polygalacturonase-inhibiting protein, and $\beta-1,3$-glucanase. Additionally, MeJA stimulated expression of genes involved in the biosynthesis of phytoalexins (phenylalanine ammonia lyase and stilbene synthase), together with an accumulation of stilbenes, which are antimicrobial compounds. A capability of promoting resistance against pathogen attack was confirmed by increased tolerance towards the fungal pathogen Erysiphe necator (powdery mildew) after treatment with MeJA. Thus, it was demonstrated that treatment with MeJA is an alternative strategy for stimulating natural defense mechanisms in $V$. vinifera, reducing the need for fungicides against powdery mildew (Belhadj et al., 2006).

It has been a few years since the first models for wound-induced signaling pathways in plants 
were proposed (Farmer and Ryan, 1992). Currently, the most widely accepted model includes the systemin peptide, bearing 18 amino acids, which is a C-terminal cleavage product of its prosytemin precursor, containing 200 amino acids (Ryan and Pearce, 2003). Systemin molecules are proficient in inducing expression of genes involved in signaling pathways leading to the release of linolenic acid, the primary precursor for the biosynthesis of JA. Jasmonic acid and systemins are able to amplify the signal induced by wounding; this is suggested to occur in vascular tissue cells. This hypothesis is based on the fact that expression of genes implicated in the biosynthesis of both prosystemin and JA was detected in vascular-bundle cells (Hause et al., 2003; Narvaez-Vasquez and Ryan, 2004; Rosahl and Feussner, 2005). Systemin was primarily considered to be a mobile molecule of systemic signaling, responsible for the induction of defense response in distal and undamaged tissues. However, recent studies suggest $\mathrm{JA}$ to be this mobile signaling molecule, as systemin would be responsible for the systemic signaling induced by wounding in tissues of damaged leaves, and not for distal signaling (Stratmann, 2003).

In tomato plants, the capacity of cells to sense and respond to JA is directly dependent on the signaling cascade initiated by prosystemin and systemin molecules. Genetic screenings aimed at identifying mutations related to suppression of prosystemin-mediated gene expression revealed 13 independent mutants named suppressed in prosystemin-mediated responses (spr). Among eight of them, four distinct genetic complementation groups were defined: namely spr-1, 2, 3, and 4, and two new alleles of the defl mutant (deficient in the accumulation of JA) were identified. Mutant plants defl, sprl and spr 2 exhibit a weak expression of proteinase inhibitors induced by systemin and wounding. Also, their ability to respond to exogenous JA treatment indicates that these mutations affect processes related to biosynthesis or accumulation of JA. This hypothesis is based on the fact that defl mutant plants are incapable of accumulating JA in response to wounding and systemin (Li et al., 2001). Various other researchers have added genetic and biochemical evidence suggesting (pro)systemin to be the fundamental regulatory molecule involved in systemic defense response mechanisms induced by wounding (Ryan, 2000; Gatehouse, 2002; Howe, 2004; Schilmiller and Howe, 2005).

Two general models were proposed to explain how the interaction between systemin and JA occurs in systemic signaling responses. In the first model, systemin produced at the wound site is translocated to distant leaves, where it activates the octadecanoid pathway (source of JA) and induces the expression of proteinase inhibitor genes. In the second model, considered an alternative, systemin would act specifically at the wound site, leading to production of JA, which in turn, would work as a mobile signaling molecule for the production of proteinase inhibitors (Schilmiller and Howe, 2005). Nevertheless, for both models to work properly, a functional signaling pathway for JA is necessary (for instance, through the CORONATINE-INSENSITIVE1 [COII] pathway), allowing recognition of the systemic signal in distal leaves. Drafting experiments utilizing jail mutants revealed that response to wounds is dependent on the production of JA in damaged leaves, but not in undamaged ones. Another interpretation suggests that systemin acts locally at the wound site, or nearby, intensifying the accumulation of JA, and consequently increasing the strength of the systemic response. Hence, this model supports the idea of systemin interacting with JA, through a positive feedback mechanism, propagating distal signaling (Ryan and Moura, 2002; Schilmiller and Howe, 2005).

The availability of Arabidopsis mutants for the JA biosynthesis pathway has given even more detail about the critical importance of JA for defense response in plants (Rosahl and Feussner, 2005). Looking at jasmonates in signaling defense pathways in Arabidopsis, McConn and co-workers (1997) made use of the triple mutant fad3-2 fad7-2 fad8 (McConn and Browse, 1996), which is deficient in the capacity to increase linolenic acid, a primary jasmonate precursor. As it produces very low levels of JA, the mutant exhibits a high mortality rate $(\sim 80 \%$ when 
compared to wild type) when attacked by Bradysia impatiens larvae. The rate of mortality was reduced to $\sim 12 \%$ when the same plants were treated exogenously with MeJA. Moreover, this mutant was incapable of accumulating transcripts of three different wound-induced genes after damage; however, exogenous treatment with MeJA induced their expression. Thus, it was demonstrated that jasmonates play a fundamental role in inducing defense mechanisms that are biologically effective, particularly for plant-insect interactions (McConn et al., 1997).

Additional studies showed that other derivatives of jasmonate also have important roles in defense responses. The Arabidopsis opr 3 mutant does not produce an isoform of the enzyme 12-oxophytodienoate reductase (12-OPR), essential for JA biosynthesis (Stintzi and Browse, 2000). Differently from JA-insensitive plants, such as the mutant coil and the triple mutant fad3-2 fad7-2 fad8, the opr 3 mutant displayed increased resistance to the insect $B$. impatiens, and the fungus $\mathrm{Al}$ ternaria brassicicola. Analysis of the transcriptional profile of opr 3 indicated that genes formerly known for JA-dependent expression after wounding are also expressed in this mutant, suggesting that cyclopentenone jasmonate derivatives can fulfill some JA tasks in vivo. Also, opr3 plants treated exogenously with OPDA are capable of robustly up-regulating the expression of a series of genes related to defense. In addition, they use cyclopentenone forms of OPDA in the regulation of gene expression cooperatively with JA, composing a fine-tuned regulation of defense genes expression, enhancing resistance against insect and fungal attack in the absence of JA (Stintzi et al., 2001).

Soybean (Glycine max) cells in suspension accumulate phytoalexins-type glyceollins in the presence of 12-OPDA. Jasmonic acid and MeJA besides the bacterial phytotoxin coronatine (a highly active compound in the defense response) presented weak activity in inducing phytoalexins when compared to 12-OPDA in cell culture (Fliegmann et al., 2003). Potato plants inoculated with the pathogen $P$. syringae pv. maculicola elicited a 15 -fold increase in the accumulation of 12-OPDA compared to JA. Infiltrated leaves, as well as intact leaves of infiltrated plants, exhibited the highest accumulation of 12-OPDA. On the other hand, accumulation of JA was observed only in leaves infiltrated with pathogen, suggesting that 12-OPDA, a primary precursor of JA biosynthesis, can play an important part in acquired systemic resistance in potato plants (Landgraf et al., 2002).

Another mechanism used in plants to further improve defense responses is based on the cross-talk between different hormonal signaling pathways, influencing not only processes related to growth and development, but also adaptation to biotic and abiotic stresses (Kazan and Manners, 2008). Generally, salicylic acid is part of defense responses against biotrophic pathogens, while JA participates in defense responses against necrotrophic pathogens and insects (Beckers and Spoel, 2006). Recent studies argue that plants, after recognition of the phytopathogen invader, are able to precisely discriminate between different defense signaling pathways, by means of synergistic and antagonistic interactions (Pieterse et al., 2001). This kind of crosstalk between different signals promotes an efficient regulatory potential, setting an alert state in plants and channeling defense towards the specific pathogen invader (Beckers and Spoel, 2006).

An example of synergistic interaction between JA, salicylic acid and ethylene was demonstrated in mutant tomato plants deficient in the accumulation of JA inoculated with the compatible pathogen Xanthomonas campestris pv. vesicatoria $(\mathrm{Xcv})$. It was observed that a JA-related primary signaling must precede the accumulation and action of ethylene and salicylic acid, suggesting a sequential and cooperative interaction between these hormones towards the development of diseases in tomato. Therefore, tomato plants would be capable of actively regulating response to disease caused by Xcv, provoking extensive cell death of own tissue (O’Donnell et al., 2003). 
Other examples of antagonistic interactions are described below. The phytopathogen $P$. syringae produces the phytotoxin coronatine as a virulent factor, which is structurally similar to the jasmonate conjugate JA-L-Ile. Coronatine is an extremely active molecule in the defense response, as it is competent to activate the JA-signaling pathway due to its structural similarity with JA-L-Ile. In the coil mutant of Arabidopsis, this activation suppresses the salicylic acid-signaling pathway, which performs a key role in the defense against this specific pathogen. This strategy leads to a delay in the defense responses, allowing the pathogen the opportunity to successfully colonize host tissues (Kunkel and Brooks, 2002; Yi et al., 2009). This same mutant shows overactivation of the PR1 gene (Pathogenesis-related1) and can accumulate high levels of salicylic acid after $P$. syringae infection, suggesting that the COII gene is a negative regulator of salicylic acid-dependent responses (Kloek et al., 2001). Arabidopsis mutant enhanced disease susceptibility4 (eds4) plants also show an interaction between the signaling pathways of salicylic acid and JA. In this case, diminished levels of salicylic acid in response to pathogen attack reflect enhanced susceptibility after $P$. syringae infection. Nonetheless, a considerable increase in the induction of gene expression dependent on JA is noted, insinuating that in wild-type Arabidopsis plants high amounts of salicylic acid can repress defense responses regulated by JA (Gupta et al., 2000).

Preston and co-workers (1999) found that salicylic acid strongly inhibits JA-dependent defense pathways in Nicotiana tabacum. Plants inoculated with tobacco mosaic virus displayed an endogenous and systemic increase in the levels of salicylic acid. Larvae of M. sexta, an herbivore known for being susceptible to JA-mediated defenses, consumed 1.7-2.7 times more tissue of plants inoculated with tobacco mosaic virus when compared to control plants. Also, it was concluded that, when inoculated with tobacco mosaic virus, $N$. tabacum plants are incapable of promoting normal defense responses induced by wounding, probably due to the inhibition of JA production, which in turn is a consequence of the high levels of systemic salicylic acid caused by tobacco mosaic virus infiltration (Preston et al., 1999).

In summary, a few relevant scenarios regarding defense response mechanisms in plants can be drawn: 1) distinct genetic studies sustain the idea that jasmonate-signaling pathways play a crucial role in defense responses induced by wounding; 2 ) damaged tissue, along with systemin, is capable of activating the expression of proteinase inhibitors, by means of a common signaling pathway requiring JA; 3) the interaction between signaling pathways of different vegetal hormones, such as salicylic acid and JA, provides plants a refined regulatory potential in response to stresses generated by pathogen attack (Rosahl and Feussner; 2005; Beckers and Spoel, 2006).

\section{Jasmonates are involved in plant reproduction}

Besides their participation in the fundamental physiological processes of development and survival, jasmonates also perform important roles related to plant reproduction. The presence of JA and MeJA has been described in anthers and pollen of three species of Camellia (Yamane et al., 1982). In early studies, JA, but not MeJA, was suggested to be the endogenous pollen germination regulator. More than 10 years later, two jasmonate compounds, $N$-[(-)-jasmonoyl]-(S)-isoleucine and $N$-[7-iso-cucurbinoyl]-(S)-isoleucine, were identified in pollen grains of Pinus mugo (Knöfel and Sembdner, 1995). The authors reinforced the idea that jasmonates play an important role in maturation and/or germination of pollen grains. Additionally, a new jasmonate ( $\mathrm{N}$-[(-)-jasmonoyl]tyramine) was identified in pollen grains of Petunia hybrida, and its structural characterization was described based on results of LC-MS-MS, GC-MS and CD assays (Miersch et al., 1998). 
The effective involvement of jasmonates in the reproductive process only became more evident when Arabidopsis mutants incapable of responding to JA were shown to have a malesterile phenotype (Table 1). Analyzing these phenotypes, it was shown that jasmonates are important not only for proper pollen development, but also have interesting functions related to stamen elongation and the correct timing of pollen release (anther dehiscence; Liechti and Farmer, 2006). Arabidopsis coil mutants were incapable of producing viable pollen grains and therefore were male-sterile (Feys et al., 1994). Genes encoding enzymes of the jasmonate biosynthetic pathway were shown to be specifically expressed in floral organs (ovaries, petals and sepals). Such floral organ-specific expression suggests that signals produced in these organs are transported to the stomium, the tissue responsible for the release of mature pollen grains (Cheong and Choi, 2003).

\begin{tabular}{|c|c|c|c|c|}
\hline Mutants & Altered phenotype & Altered process & Affected gene & References \\
\hline coil & male-sterile & proteolysis via SCF & $\mathrm{COIl,} \mathrm{F-box} \mathrm{protein}$ & Feys et al., 1994 \\
\hline ddel & male-sterile & OPDA conversion & $O P R 3$ & Sanders et al., 2000 \\
\hline cevl & constitutive JA response & cell wall synthesis & $\mathrm{CeS3}$ & Ellis and Turner, 2001 \\
\hline dadl & male-sterile & $\alpha$-linolenic acid ( $\alpha$-LeA) liberation & Phospholipase A1 & Ishiguro et al., 2001 \\
\hline$d d e 2-2$ & male-sterile & OPDA conversion & $\operatorname{AOS}$ & von Malek et al., 2002 \\
\hline jail & female-sterile & seed maturation control & COIl, F-box protein & Li et al., 2004 \\
\hline jarl & JA insensitive & JA conjugation & $J A R I$, JA amino acid synthetase & Staswick and Tiryaki, 2004 \\
\hline acxl & IBA resistant & $\beta$-oxidation & $A C X 1$ & Adham et al., 2005 \\
\hline$c t s / p x a$ & JA deficient & $\beta$-oxidation & COMATOSE/PXAI & Theodoulou et al., 2005 \\
\hline
\end{tabular}

The Arabidopsis mutant delayed dehiscence 1 (dde1), which has a T-DNA inserted in the gene encoding for the enzyme 12-OPR, showed a delayed dehiscence of the anther, resulting in inefficient fertilization. Exogenous JA application was capable of restoring the wild-type phenotype, allowing the originally male-sterile mutant plants to produce seeds. In situ hybridization experiments have shown that in later stages of flower development, accumulation of $D D E 1$ mRNA occurs specifically in pistil tissues, petals, and anther filaments. In contrast, no DDE1 mRNA was detected in the stomium, the cells of the anther septum that are directly involved in the pollen release. These results suggest that JA-dependent signaling has an important role in the timing of anther dehiscence (Sanders et al., 2000). Additional Arabidopsis mutants with a male-sterile phenotype were shown to occur in enzymes responsible for the conversion of $\alpha$-LeA to JA, important steps of the jasmonate biosynthesis pathway (Stintzi and Browse, 2000; von Malek et al., 2002). One of the mutations was shown to occur in a gene for 12-OPR, which was denominated OPR3 (Stintzi and Browse, 2000). Mutant plants (opr3) were found to be sterile, a phenotype that was overcome by exogenous application of JA, but not OPDA, the immediate precursor of JA. Therefore, it was suggested that JA itself is the signaling jasmonate capable of inducing and coordinating the elongation of the anther filament, the opening of the stomium at anthesis, and the production of viable pollen in Arabidopsis (Stintzi and Browse, 2000). Recently, stamens from opr3 Arabidopsis mutants were used for a large-scale gene expression analysis following JA treatment. This analysis allowed the identification of 13 transcription factors involved in stamen maturation, such as MYB21 and MYB24, which are JA-inducible and were shown to intermediate the response to jasmonates during stamen development (Mandaokar et al., 2006).

A screening for Arabidopsis mutants with fertility problems in a transposontagged population allowed identification of the delayed dehiscence2-2 (dde2-2) mutant. 
Mutant dde2-2 plants exhibited problems related to elongation of the anther filament, anther dehiscence and they were male-sterile. The wild-type phenotype was recovered by exogenous application of MeJA, indicating that the mutation directly affected the jasmonate biosynthesis pathway. Additional studies of gene expression and complementation analysis showed that dde2-2 male sterility was caused by interruption of the gene sequence encoding AOS, one of the main enzymes of the JA biosynthesis pathway (von Malek et al., 2002).

Similar to the dde2-2 mutants, the mutation defective in anther dehiscencel (dad1) produces a male-sterile phenotype in Arabidopsis. Mutant dad1 plants show problems related to anther dehiscence, pollen grain maturation and flower opening (anthesis). Exogenous application of JA or $\alpha$-LeA rescued the mutant phenotype, which was consistent with reduced JA levels in floral buds of $d a d 1$ mutants. Furthermore, the fusion protein DAD1-GFP was shown to be localized in the chloroplasts. It has been suggested that the DAD1 gene encodes the chloroplast-specific phospholipase A1, the enzyme responsible for catalyzing the formation of $\alpha$-LeA, the primordial precursor of the JA biosynthesis pathway (Ishiguro et al., 2001).

Jasmonates seem to have a general importance in regulating reproductive processes in plants, not restricted to Arabidopsis. In Solanaceae, mutants displaying famalesterile phenotype were obtained and were found to have alterations in important genes of the jasmonate biosynthesis pathway. Tomato jail mutant (Table 1), which is defective in JA signaling, exhibits reduced pollen viability and sterility, which is caused by a defect in the maternal control of seed maturation, associated with the loss of accumulation of JA-regulated proteinase-inhibitor proteins in reproductive tissues (Li et al., 2004). Interestingly, in contrast to what occurs in Arabidopsis, the work done with tomato plants suggests that in this species jasmonates mediate a female-sterile phenotype (Li et al., 2001). It has been demonstrated that the jasmonates JA, 12-OPDA, and the JA-L-Ile conjugate accumulate at levels twice as high in the floral tissues than in leaves. Furthermore, results from histochemical analysis showed a great accumulation of the allene oxide cyclase protein specifically in ovules, stylar transmitting tissue, and vascular bundles of the floral receptacles, implicating JA biosynthesis pathway in the regulation of floral/pistil development in tomato plants (Hause et al., 2000).

Recent studies performed with monocot plants of great economic importance, such as corn and rice, demonstrated the involvement of jasmonates in the development of the male reproductive structure. In corn plants (Zea mays), formation of the male flower, the tassel, was affected in tasselseed mutants ( $t s 1$ and $t s 2$ ), causing the development of female flowers on the tassel (Browse, 2009b). Cloning of $t s 1$ has revealed that the TS1 protein is a 13-lipoxygenase, an enzyme involved in jasmonate synthesis. The JA concentration measured at the tassels of ts 1 homozygote plants was $1 / 10$ of that of the wild-type tassels. However, treatment of $t s 1$ mutant tassels with $1 \mathrm{mM} \mathrm{JA}$ only partly recovered the wild-type phenotype. These results demonstrate that jasmonate is an essential signal for determining male identity in the maize tassel (Browse, 2009b).

The P0491E01 gene identified in rice encodes a protein with high similarity to DAD1 (DEFECTIVE IN ANTHER DEHISCENCE1), which is involved in JA biosynthesis in Arabidopsis. RNAi P0491E01 rice transgenic plants (Oryza sativa japonica) were found to be male-sterile or exhibited reduced fertility. Cytological studies performed in the male-sterile transgenic plants showed normal anther development at the initial stages, 
but microspore development into mature pollen grains was impaired. Moreover, RT-PCR experiments showed that expression of the P0491E01 gene was significantly reduced in transgenic plants, suggesting that jasmonates fulfill an important role in later stages of anther development (Yu et al., 2006).

The data presented above clearly show the importance of adequate jasmonate biosynthesis and signaling in the accomplishment of the reproductive process and the formation of the male and/or female sexual structures in monocot and dicot flowers. Obtaining new mutants for other important enzymes of the jasmonate biosynthetic pathway will certainly increase our understanding of the complex physiological mechanisms taking place during plant reproduction.

\section{Genes of the jasmonate biosynthesis pathway are expressed in Nicotiana tabacum pistils}

The study developed in our laboratory aims to identify and characterize genes preferentially or specifically expressed in the female reproductive organ of the plant, the pistil, and to study their function in pistil development and/or pollen-pistil interaction. As a model we use pistils of Nicotiana tabacum cv. Petit Havana SR-1 (Solanaceae), which are composed of stigma, style and ovary. To perform large-scale analysis of genes expressed in the tobacco pistil, we constructed and sequenced a stigma/style cDNA library generating the TOBEST database, which has been analyzed and described in a recent paper (Quiapim et al., 2009).

In a previous research carried out in our laboratory, differential screening of a tobacco stigma/style cDNA library resulted in the identification of a pistil-specific cDNA clone, encoding a protein with high similarity to methyltransferases of the SABATH family (Ângelo, 2001; Avanci NC, Calixto CPG, Ângelo PCS, Molfetta-Machado JB, et al., unpublished results). The experiments show that the enzyme encoded by the cDNA is capable of synthetizing MeJA (Avanci NC, Calixto CPG, Ângelo PCS, Molfetta-Machado JB, et al., unpublished results), raising a question about its function in the tobacco flowers.

To obtain a better understanding of the importance of jasmonates in tobacco pistils, we searched the TOBEST database for sequences encoding the enzymes of the jasmonate biosynthetic pathway. The Arabidopsis amino acid sequences for LOX (acession number: At1G17420.1), AOS (accession number: At5G42650.1), allene oxide cyclase (accession number: At1G13280.1), and 12-OPR (accession number: At1G09400.1) were used to perform a TBlastN in the TOBEST database. The tobacco sequences identified were then analyzed by the BLAST program in the National Center of Biotechnology Information (NCBI) database (http://blast.ncbi.nlm.nih.gov/Blast.cgi, accessed November 25,2009 ) to confirm their annotation as enzymes of the jasmonate biosynthesis pathway. The results are shown in Figure 3. We were able to find cDNA clones corresponding to LOX (TOBS114G06), allene oxide cyclase (TOBS040E09) and 12-OPR (TOBS076D02). However, we did not find a clone with significant similarity to AOS. The sequences identified for the other three enzymes belong to singlets (present as a unique clone in the TOBEST cDNA library), which points to a low expression level for the genes in this pathway. Therefore, the absence of the AOS sequence in TOBEST may have happened by chance. Alternatively, AOS absence may indicate strict regulation of the JA biosynthesis in tobacco pistils, occurring at this crucial step of the pathway, as already suggested (Laudert and Weiler, 1998). 


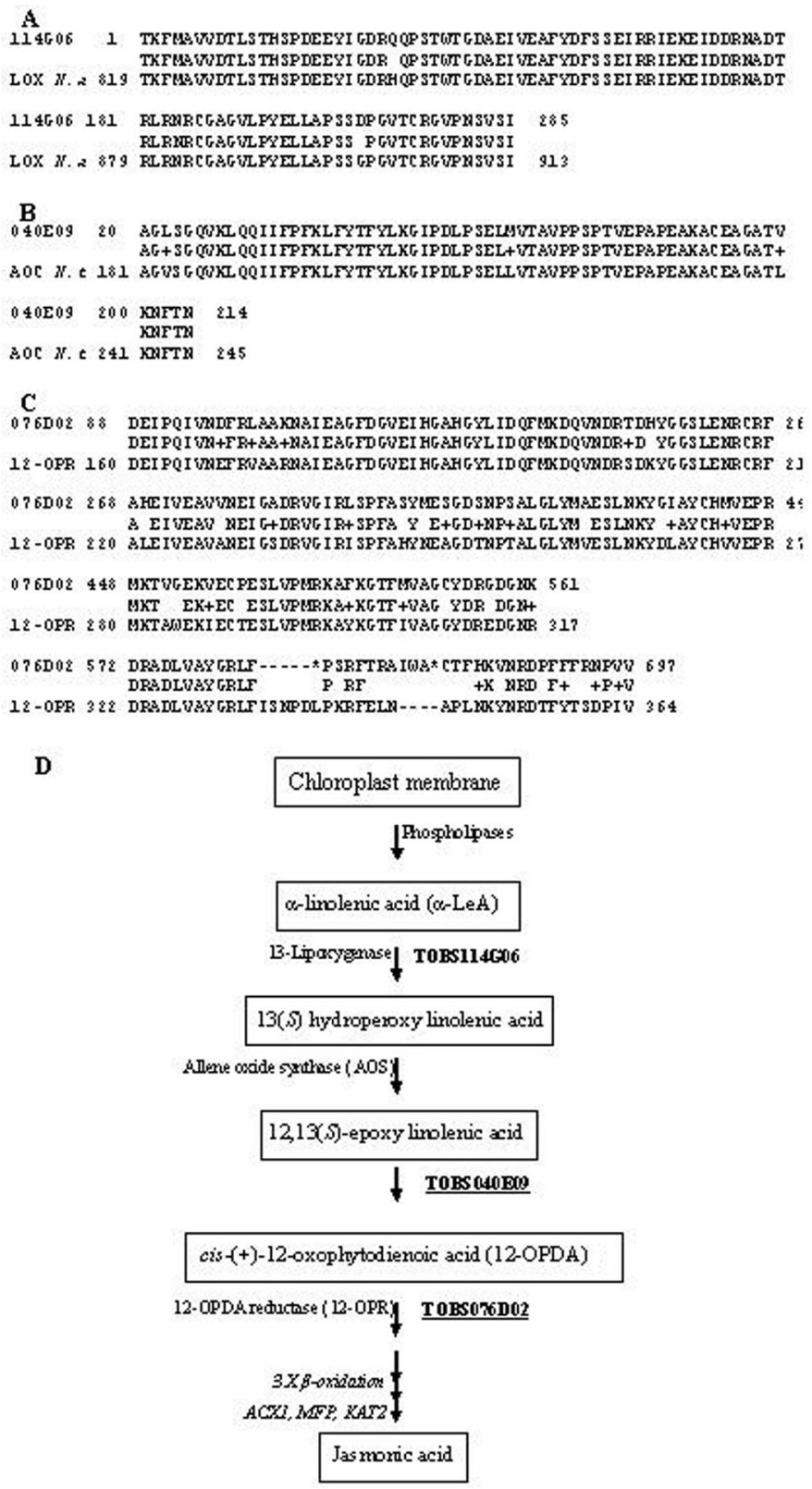

Figure 3. Analysis of the main enzymes of the jasmonate biosynthesis pathway identified in the TOBEST database. A. Alignment of the lipoxygenase (LOX) from Nicotiana attenuata with the deduced amino acid sequence of the TOBS114G06 clone (97\% identity). B. Alignment of the allene oxide cyclase from $N$. tabacum with the deduced amino acid sequence of the TOBS040E09 clone (95\% identity). C. Alignment of the 12-OPDA reductase (12-OPR) from Ricinum communis with the deduced amino acid sequence of the TOBS076D02 clone (83\% identity). D. Simplified model of jasmonic acid biosynthesis pathway. The TOBEST clones are shown at the right side of the figure and are highlighted in bold and underlined, side-by-side with their corresponding enzymes. 
The representation of LOX, allene oxide cyclase and 12-OPR, in addition to a pistilspecific JA methyltransferase (Avanci NC, Calixto CPG, Ângelo PCS, Molfetta-Machado JB, et al., unpublished results), strongly supports the involvement of jasmonates in the developmental and physiological processes taking place in the tobacco female reproductive organ. The contribution of jasmonates to the proper development of the female reproductive organ of the tomato, another Solanaceae species, has already been reported (Hause et al., 2000; Li et al., 2001, 2004). Conversely, in Arabidopsis (Brassicaceae) jasmonates are involved in the development of the male reproductive organs (Sanders et al., 2000; Ishiguro et al., 2001; von Malek et al., 2002). Despite the differences in female or male reproductive development contributions, it is unquestionable that jasmonates are key hormones for the plant reproduction process.

\section{CONCLUDING REMARKS}

The description and characterization of various mutants, arising from different collections, help in our understanding of the biosynthesis, regulation, and signaling mechanisms of jasmonates. Responses to biotic and abiotic stresses, as well as processes related to plant development and reproduction, are greatly impaired when jasmonate-dependent gene expression is affected. Furthermore, JA and MeJA, as well as jasmonate-intermediates, play crucial roles in defense responses. In conclusion, it is evident that jasmonates, which were previously associated mainly with defense responses, have multiple functions, including an important role in plant reproduction.

\section{ACKNOWLEDGMENTS}

The authors are grateful to Paulo Rosa Júnior for hand-drawing Figure 1. Research supported by grants from Fundação de Amparo à Pesquisa do Estado de São Paulo - Brazil (FAPESP \#0654431-9) and from Conselho Nacional de Desenvolvimento Científico e Tecnológico (CNPq) - Brazil. N.C. Avanci was supported by a fellowship from Coordenação de Aperfeiçoamento de Pessoal de Nível Superior (CAPES) and D.D. Luche by a fellowship from CNPq (Fixação de Recursos Humanos do CNPq - Nível 3B) for Invent Biotecnologia company. G.H. Goldman and M.H.S. Goldman are indebted to CNPq for their research fellowships.

\section{REFERENCES}

Adham AR, Zolman BK, Millius A and Bartel B (2005). Mutations in Arabidopsis acyl-CoA oxidase genes reveal distinct and overlapping roles in beta-oxidation. Plant J. 41: 859-874.

Ângelo PCS (2001). Identificação e Análise de Genes Expressos Exclusivamente no Pistilo de Nicotiana tabacum L. Doctoral thesis, Faculdade de Medicina de Ribeirão Preto, Ribeirão Preto.

Arimura G, Kost C and Boland W (2005). Herbivore-induced, indirect plant defences. Biochim. Biophys. Acta 1734: 91-111. Beckers GJ and Spoel SH (2006). Fine-tuning plant defence signalling: Salicylate versus Jasmonate. Plant Biol. 8: 1-10.

Belhadj A, Saigne C, Telef N, Cluzet S, et al. (2006). Methyl jasmonate induces defense responses in grapevine and triggers protection against Erysiphe necator. J. Agric. Food Chem. 54: 9119-9125.

Berger S (2002). Jasmonate-related mutants of Arabidopsis as tools for studying stress signaling. Planta 214: 497-504.

Boter M, Ruiz-Rivero O, Abdeen A and Prat S (2004). Conserved MYC transcription factors play a key role in jasmonate signaling both in tomato and Arabidopsis. Genes Dev. 18: 1577-1591.

Browse J (2009a). Jasmonate passes muster: a receptor and targets for the defense hormone. Annu. Rev. Plant Biol. 60: 183-205. Browse J (2009b). Jasmonate: preventing the maize tassel from getting in touch with his feminine side. Sci. Signal. 2: e9. Chen H, Jones AD and Howe GA (2006). Constitutive activation of the jasmonate signaling pathway enhances the 
production of secondary metabolites in tomato. FEBS Lett. 580: 2540-2546.

Cheong JJ and Choi YD (2003). Methyl jasmonate as a vital substance in plants. Trends Genet. 19: 409-413.

Chini A, Fonseca S, Fernandez G, Adie B, et al. (2007). The JAZ family of repressors is the missing link in jasmonate signalling. Nature 448: 666-671.

Creelman RA and Mullet JE (1995). Jasmonic acid distribution and action in plants: regulation during development and response to biotic and abiotic stress. Proc. Natl. Acad. Sci. U. S. A. 92: 4114-4119.

Dathe W, Rönsch H, Preiss A, Schade W, et al. (1981). Endogenous plant hormones of the broad bean, Vicia faba L. (-)-jasmonic acid, a plant growth inhibitor in pericarp. Planta 153: 530-535.

Delker C, Stenzel I, Hause B, Miersch O, et al. (2006). Jasmonate biosynthesis in Arabidopsis thaliana - enzymes, products, regulation. Plant Biol. 8: 297-306.

Demole E, Lederer E and Mercier O (1962). Isolement et détermination dela structure du jasmonate de méthyle, constituant odorant caractéristique de l'essencede jasmin. Helv. Chim. Acta 45: 675-685.

Devoto A, Nieto-Rostro M, Xie D, Ellis C, et al. (2002). COI1 links jasmonate signalling and fertility to the SCF ubiquitinligase complex in Arabidopsis. Plant J. 32: 457-466.

Ellis C and Turner JG (2001). The Arabidopsis mutant cev1 has constitutively active jasmonate and ethylene signal pathways and enhanced resistance to pathogens. Plant Cell 13: 1025-1033.

Ellis C, Karafyllidis I, Wasternack C and Turner JG (2002). The Arabidopsis mutant cev1 links cell wall signaling to jasmonate and ethylene responses. Plant Cell 14: 1557-1566.

Farmer EE and Ryan CA (1992). Octadecanoid precursors of jasmonic acid activate the synthesis of wound-inducible proteinase inhibitors. Plant Cell 4: 129-134.

Farmer EE, Johnson RR and Ryan CA (1992). Regulation of expression of proteinase inhibitor genes by methyl jasmonate and jasmonic acid. Plant Physiol. 98: 995-1002.

Farmer EE, Almeras E and Krishnamurthy V (2003). Jasmonates and related oxylipins in plant responses to pathogenesis and herbivory. Curr. Opin. Plant Biol. 6: 372-378.

Feys B, Benedetti CE, Penfold CN and Turner JG (1994). Arabidopsis mutants selected for resistance to the phytotoxin coronatine are male sterile, insensitive to methyl jasmonate, and resistant to a bacterial pathogen. Plant Cell 6: 751-759.

Fliegmann J, Schuler G, Boland W, Ebel J, et al. (2003). The role of octadecanoids and functional mimics in soybean defense responses. Biol. Chem. 384: 437-446.

Fonseca S, Chini A, Hamberg M, Adie B, et al. (2009). (+)-7-iso-Jasmonoyl-L-isoleucine is the endogenous bioactive jasmonate. Nat. Chem. Biol. 5: 344-350.

Gatehouse JA (2002). Plant resistance towards insect herbivores: a dynamic interaction. New Phytol. 156: 145-169.

Green TR and Ryan CA (1972). Wound-induced proteinase inhibitor in plant leaves: a possible defense mechanism against insects. Science 175: 776-777.

Gupta V, Willits MG and Glazebrook J (2000). Arabidopsis thaliana EDS4 contributes to salicylic acid (SA)-dependent expression of defense responses: evidence for inhibition of jasmonic acid signaling by SA. Mol. Plant Microbe Interact. 13: 503-511.

Halitschke R and Baldwin IT (2003). Antisense LOX expression increases herbivore performance by decreasing defense responses and inhibiting growth-related transcriptional reorganization in Nicotiana attenuata. Plant J. 36: 794-807.

Halitschke T and Baldwin IT (2004). Jasmonates and related compounds in plant-insect interactions. J. Plant Growth Regul. 23: 238-245.

Hause B, Stenzel I, Miersch O, Maucher H, et al. (2000). Tissue-specific oxylipin signature of tomato flowers: allene oxide cyclase is highly expressed in distinct flower organs and vascular bundles. Plant J. 24: 113-126.

Hause B, Hause G, Kutter C, Miersch O, et al. (2003). Enzymes of jasmonate biosynthesis occur in tomato sieve elements. Plant Cell Physiol. 44: 643-648.

Howe GA (2004). Jasmonates as signals in the wound response. J. Plant Growth Regul. 23: 223-237.

Howe GA, Lightner J, Browse J and Ryan CA (1996). An octadecanoid pathway mutant (JL5) of tomato is compromised in signaling for defense against insect attack. Plant Cell 8: 2067-2077.

Ishiguro S, Kawai-Oda A, Ueda J, Nishida I, et al. (2001). The DEFECTIVE IN ANTHER DEHISCIENCE gene encodes a novel phospholipase A1 catalyzing the initial step of jasmonic acid biosynthesis, which synchronizes pollen maturation, anther dehiscence, and flower opening in Arabidopsis. Plant Cell 13: 2191-2209.

Kazan K and Manners JM (2008). Jasmonate signaling: toward an integrated view. Plant Physiol. 146: 1459-1468.

Kessler A and Baldwin IT (2002). Plant responses to insect herbivory: the emerging molecular analysis. Annu. Rev. Plant Biol. 53: 299-328.

Kloek AP, Verbsky ML, Sharma SB, Schoelz JE, et al. (2001). Resistance to Pseudomonas syringae conferred by an Arabidopsis thaliana coronatine-insensitive (coi1) mutation occurs through two distinct mechanisms. Plant J. 26: 509-522.

Knöfel HD and Sembdner G (1995). Jasmonates from pine pollen. Phytochemistry 38: 569-571. 
Koda Y (1997). Possible involvement of jasmonates in various morphogenic events. Physiol. Plant. 100: 639-646.

Kolomiets MV, Chen H, Gladon RJ, Braun EJ, et al. (2000). A leaf lipoxygenase of potato induced specifically by pathogen infection. Plant Physiol. 124: 1121-1130.

Kunkel BN and Brooks DM (2002). Cross talk between signaling pathways in pathogen defense. Curr. Opin. Plant Biol. 5: 325-331.

Landgraf P, Feussner I, Hunger A, Scheel D, et al. (2002). Systemic accumulation of 12-oxo-phytodienoic acid in SARinduced potato plants. Eur. J. Plant Pathol. 108: 279-283.

Laudert D and Weiler EW (1998). Allene oxide synthase: a major control point in Arabidopsis thaliana octadecanoid signalling. Plant J. 15: 675-684.

Li L, Li C and Howe GA (2001). Genetic analysis of wound signaling in tomato. Evidence for a dual role of jasmonic acid in defense and female fertility. Plant Physiol. 127: 1414-1417.

Li L, Zhao Y, McCaig BC, Wingerd BA, et al. (2004). The tomato homolog of CORONATINE-INSENSITIVE1 is required for the maternal control of seed maturation, jasmonate-signaled defense responses, and glandular trichome development. Plant Cell 16: 126-143.

Liechti R and Farmer EE (2006). Jasmonate biochemical pathway. Sci. STKE 322: 1-3.

Lorenzo O and Solano R (2005). Molecular players regulating the jasmonate signalling network. Curr. Opin. Plant Biol. 8: $532-540$.

Lorenzo O, Chico JM, Sanchez-Serrano JJ and Solano R (2004). JASMONATE-INSENSITIVE1 encodes a MYC transcription factor essential to discriminate between different jasmonate-regulated defense responses in Arabidopsis. Plant Cell 16: 1938-1950.

Maciejewska B and Kopcewicz J (2003). Inhibitory effect of methyl jasmonate on flowering and elongation growth in pharbitis nil. J. Plant Growth Regul. 21: 216-223.

Mandaokar A, Thines B, Shin B, Lange BM, et al. (2006). Transcriptional regulators of stamen development in Arabidopsis identified by transcriptional profiling. Plant J. 46: 984-1008.

McConn M and Browse J (1996). The critical requirement for linolenic acid in pollen development, not photosynthesis, in an Arabidopsis mutant. Plant Cell 8: 403-416.

McConn M, Creelman RA, Bell E, Mullet JE, et al. (1997). Jasmonate is essential for insect defense in Arabidopsis. Proc. Natl. Acad. Sci. U. S. A. 94: 5473-5477.

Mei C, Qi M, Sheng G and Yang Y (2006). Inducible overexpression of a rice allene oxide synthase gene increases the endogenous jasmonic acid level, PR gene expression, and host resistance to fungal infection. Mol. Plant Microbe Interact. 19: 1127-1137.

Miersch O, Knöfel HD, Schmidt J, Kramell R, et al. (1998). A jasmonic acid conjugate, N-[(-)-jasmonoyl]-tyramine, from Petunia pollen. Phytochemistry 47: 327-329.

Narvaez-Vasquez J and Ryan CA (2004). The cellular localization of prosystemin: a functional role for phloem parenchyma in systemic wound signaling. Planta 218: 360-369.

Norastehnia A and Asghari MN (2006). Effects of methyl jasmonate on the enzymatic antioxidant defense system in maize seedlings subjected to Paraquat. Asian J. Plant Sci. 5: 17-23.

O’Donnell PJ, Schmelz E, Block A, Miersch O, et al. (2003). Multiple hormones act sequentially to mediate a susceptible tomato pathogen defense response. Plant Physiol. 133: 1181-1189.

Pieterse CMJ, Ton J and Van Loon LC (2001). Cross-talk between plant defence signalling pathways: boost or burden? AgBiotechNet 3 ABN 068: 1-8. Available at [www.agbiotechnet.com].

Preston CA, Lewandowski C, Enyedi AJ and Baldwin IT (1999). Tobacco mosaic virus inoculation inhibits woundinduced jasmonic acid-mediated responses within but not between plants. Planta 209: 87-95.

Quiapim AC, Brito MS, Bernardes LA, Dasilva I, et al. (2009). Analysis of the Nicotiana tabacum stigma/style transcriptome reveals gene expression differences between wet and dry stigma species. Plant Physiol. 149: 1211-1230.

Rickauer M, Brodschelm W, Bottin A, Véronési C, et al. (1997). The jasmonate pathway is involved differentially in the regulation of different defence responses in tobacco cells. Planta 202: 155-162.

Rosahl S and Feussner I (2005). Oxylipins. In: Plant Lipids: Biology, Utilization and Manipulation (Murphy DJ, ed.). Blackwell Publishing Ltd./CRC Press, Oxford and Boca Raton, 329-354.

Ryan CA (2000). The systemin signaling pathway: differential activation of plant defensive genes. Biochim. Biophys. Acta 1477: 112-121.

Ryan CA and Moura DS (2002). Systemic wound signaling in plants: a new perception. Proc. Natl. Acad. Sci. U. S. A. 99: 6519-6520.

Ryan CA and Pearce G (2003). Systemins: a functionally defined family of peptide signals that regulate defensive genes in Solanaceae species. Proc. Natl. Acad. Sci. U. S. A. 100 (Suppl 2): 14577-14580.

Sanchez-Serrano J, Schmidt R, Schell J and Willmitzer L (1986). Nucleotide sequence of proteinase inhibitor II encoding cDNA of potato (Solanum tuberosum) and its mode of expression. Mol. Gen. Genet. 203: 15-20.

Genetics and Molecular Research 9 (1): 484-505 (2010)

CFUNPEC-RP www.funpecrp.com.br 
Sanders PM, Lee PY, Biesgen C, Boone JD, et al. (2000). The Arabidopsis DELAYED DEHISCENCE1 gene encodes an enzyme in the jasmonic acid synthesis pathway. Plant Cell 12: 1041-1061.

Scheel D (1998). Resistance response physiology and signal transduction. Curr. Opin. Plant Biol. 1: 305-310.

Schilmiller AL and Howe GA (2005). Systemic signaling in the wound response. Curr. Opin. Plant Biol. 8: 369-377.

Sembdner G and Parthier B (1993). The biochemistry and the physiological and molecular actions of jasmonates. Annu. Rev. Plant Physiol. Plant Mol. Biol. 44: 469-489.

Seo HS, Song JT, Cheong JJ, Lee YH, et al. (2001). Jasmonic acid carboxyl methyltransferase: a key enzyme for jasmonate-regulated plant responses. Proc. Natl. Acad. Sci. U. S. A. 98: 4788-4793.

Song JT, Seo HS, Song SI, Lee JS, et al. (2000). NTR1 encodes a floral nectary-specific gene in Brassica campestris L. ssp. pekinensis. Plant Mol. Biol. 42: 647-655.

Staswick PE and Tiryaki I (2004). The oxylipin signal jasmonic acid is activated by an enzyme that conjugates it to isoleucine in Arabidopsis. Plant Cell 16: 2117-2127.

Staswick PE, Su W and Howell SH (1992). Methyl jasmonate inhibition of root growth and induction of a leaf protein are decreased in an Arabidopsis thaliana mutant. Proc. Natl. Acad. Sci. U. S. A. 89: 6837-6840.

Staswick PE, Yuen GY and Lehman CC (1998). Jasmonate signaling mutants of Arabidopsis are susceptible to the soil fungus Pythium irregulare. Plant J. 15: 747-754.

Stintzi A and Browse J (2000). The Arabidopsis male-sterile mutant, opr3, lacks the 12-oxophytodienoic acid reductase required for jasmonate synthesis. Proc. Natl. Acad. Sci. U. S. A. 97: 10625-10630.

Stintzi A, Weber H, Reymond P, Browse J, et al. (2001). Plant defense in the absence of jasmonic acid: the role of cyclopentenones. Proc. Natl. Acad. Sci. U. S. A. 98: 12837-12842.

Stratmann JW (2003). Long distance run in the wound response - jasmonic acid is pulling ahead. Trends Plant Sci. 8: 247-250.

Theodoulou FL, Job K, Slocombe SP, Footitt S, et al. (2005). Jasmonic acid levels are reduced in COMATOSE ATPbinding cassette transporter mutants. Implications for transport of jasmonate precursors into peroxisomes. Plant Physiol. 137: 835-840.

Thines B, Katsir L, Melotto M, Niu Y, et al. (2007). JAZ repressor proteins are targets of the SCF (COI1) complex during jasmonate signalling. Nature 448: 661-665.

Ueda J and Kato J (1980). Isolation and identification of a senescence-promoting substance from wormwood (Artemisia absinthium L.). Plant Physiol. 66: 246-249.

Vick BA and Zimmerman DC (1984). Biosynthesis of jasmonic acid by several plant species. Plant Physiol. 75: 458-461. von Malek B, van der Graaff E, Schneitz K and Keller B (2002). The Arabidopsis male-sterile mutant dde2-2 is defective in the ALLENE OXIDE SYNTHASE gene encoding one of the key enzymes of the jasmonic acid biosynthesis pathway. Planta 216: 187-192.

Wasternack C (2007). Jasmonates: an update on biosynthesis, signal transduction and action in plant stress response, growth and development. Ann. Bot. 100: 681-697.

Wasternack C and Hause B (2002). Jasmonates and octadecanoids: signals in plant stress responses and development. Prog. Nucleic Acid Res. Mol. Biol. 72: 165-221.

Xie DX, Feys BF, James S, Nieto-Rostro M, et al. (1998). COI1: an Arabidopsis gene required for jasmonate-regulated defense and fertility. Science 280: 1091-1094.

Xu L, Liu F, Lechner E, Genschik P, et al. (2002). The SCF(COI1) ubiquitin-ligase complexes are required for jasmonate response in Arabidopsis. Plant Cell 14: 1919-1935.

Xue R and Zhang B (2007). Increased endogenous methyl jasmonate altered leaf and root development in transgenic soybean plants. J. Genet. Genomics 34: 339-346.

Yamane H, Abe H and Takahashi N (1982). Jasmonic acid and methyl jasmonate in pollens and anthers of three Camellia species. Plant Cell Physiol. 23: 1125-1127.

Yi H, Preuss ML and Jez JM (2009). The devil (and an active jasmonate hormone) is in the details. Nat. Chem. Biol. 5: 273-274.

Yu ZQ, Zhu J, Gao JF and Yang ZN (2006). Functional analysis of rice P0491E01 gene regulating anther development. Fen. Zi. Xi. Bao. Sheng Wu Xue. Bao. 39: 467-472. 